Pathophysiology

of Haemostasis and Thrombosis
Pathophysiol Haemost Thromb 2005;34(suppl 1):18-24

DOI: $\underline{10.1159 / 000083080}$

\title{
The Role of Ximelagatran in the Treatment of Venous Thromboembolism
}

\author{
Sam Schulman \\ Department of Haematology, Karolinska University Hospital, Stockholm, Sweden, and HHS - General Hospital, \\ Hamilton, Canada
}

\section{Key Words}

Ximelagatran - Oral direct thrombin inhibitors . VTE treatment and secondary prevention - New anticoagulants - THRIVE trials · Oral vitamin $\mathrm{K}$ antagonists

\begin{abstract}
Clinical-based evidence demonstrates that long-term oral anticoagulant therapy with the vitamin $\mathrm{K}$ antagonists is highly effective for the secondary prevention of venous thromboembolism (VTE). However, owing to fear of bleeding complications and the inconvenience of coagulation monitoring, many patients do not receive the required duration of treatment. This can lead to a high incidence of recurrent VTE events and has prompted the evaluation of alternative treatment strategies and the development of new anticoagulants for VTE management. For patient groups in which it is particularly difficult to maintain the target intensity of anticoagulation, low-molecular-weight heparin (LMWH) has been found to significantly reduce the risk of recurrent VTE without increasing bleeding risk. The parenteral administration of $\mathrm{LMWH}$, however, is a drawback for long-term use in the outpatient setting. Long-term warfarin use at a lower intensity (international normalized ratio [INR] 1.5-2.0) has also been assessed as a possible strategy to reduce bleeding complications and the need for monitoring, but
\end{abstract}

(C) 2005 S. Karger AG, Basel $1424-8832 / 05 / 0347-0018 \$ 22.00 / 0$

Fax +4161306 1234 E-Mail karger@karger.ch www.karger.com www.karger.com/pht results were disappointing when compared with conventional-intensity warfarin (INR 2.0-3.0). New therapies in development that may potentially offer a more favourable benefit-risk profile and greater consistency and predictability of response include the synthetic pentasaccharides, fondaparinux and idraparinux. These parenterally administered indirect factor Xa inhibitors have a predictable pharmacokinetic profile, allowing use without coagulation monitoring. Fondaparinux to date has only been evaluated in the initial treatment (5-7 days) of symptomatic deep vein thrombosis. In contrast, idraparinux, with its longer half-life (80 h) allowing onceweekly parenteral dosing, has the potential for long-term treatment and is currently being assessed in phase III trials for the secondary prevention of VTE. Currently, the most promising new therapeutic option is the first of the oral direct thrombin inhibitors, ximelagatran. The THRombin Inhibitor in VEnous thromboembolism (THRIVE) clinical trial programme has demonstrated that this agent is as effective as standard therapy for the acute treatment (THRIVE Treatment) and secondary prevention (THRIVE III) of VTE events and is well tolerated when used for 6 months or over extended periods up to 1.5 years. Furthermore, with oral administration, fixed dosing and no requirement for anticoagulation monitoring, ximelagatran has the potential to facilitate optimal use and duration of VTE treatment by overcoming the limitations of current agents.

Copyright $\odot 2005$ S. Karger AG, Basel

Dr. Sam Schulman

HHS - General Hospital

237 Barton St. East

Hamilton, Ont. L8L 2X2 (Canada)

Tel. +1 905528 9946, Fax +1905 521 1551, E-Mail schulms@mcmaster.ca 


\section{Introduction}

The optimal approach to the management of venous thromboembolism (VTE) remains a matter for debate. Therapy for idiopathic VTE typically includes a 5- to 7day course of low-molecular-weight heparin (LMWH) followed by a minimum of 3 months of oral anticoagulation therapy with dose-adjusted warfarin to achieve an international normalized ratio (INR) between 2.0 and 3.0. After cessation of anticoagulation therapy, however, recurrent venous thrombosis is a major clinical problem with rates of $6-9 \% /$ year $[1,2]$. The risk of recurrent thrombosis after discontinuation of anticoagulant therapy depends largely on underlying risk factors being temporary or permanent, and this in turn will influence the duration of secondary prophylaxis required [3]. For example, patients with ongoing risk factors, such as thrombophilia or metastatic cancer, require prolonged anticoagulation for 6 months or longer, whereas for patients with transient risk factors, such as surgery, a shorter duration of approximately 6 weeks of postoperative therapy may be sufficient [3].

Clinical trials have demonstrated that in patients with a first episode of VTE, 6 months of prophylactic oral anticoagulation led to a lower recurrence rate than treatment lasting for 6 weeks [4], and in patients with recurrent episodes, indefinite oral anticoagulation significantly reduced the rate of recurrence over 4 years compared with 6 months of treatment [5]. Unfortunately, clinical studies have also demonstrated that indefinite therapy with vitamin $\mathrm{K}$ antagonists (VKAs), such as warfarin, carries a substantial risk of major haemorrhage, with rates of major bleeding episodes ranging from 2 to $4 \%$ annually, or from 5 to $9 \%$ in cohort studies with unselected patients [6-8]. Thus, the presence of risk factors for bleeding during anticoagulation therapy - including age, previous stroke, recent myocardial infarction, concomitant renal failure, anaemia or diabetes $[9,10]$ - must be weighed against the risk of recurrence for each individual when deciding on duration of anticoagulation therapy. The American College of Chest Physicians guidelines recommend at least 6 months of treatment for secondary prophylaxis in patients with idiopathic VTE [3]. However, these recommendations are rarely adhered to, owing to the fear of bleeding complications and the numerous other inherent limitations of current anticoagulants (described in detail by Giancarlo Agnelli in this supplement) [11].

The unfavourable benefit-risk ratio of warfarin for long-term management has prompted the evaluation of alternative treatment strategies and the pursuit of novel anticoagulants with improved benefit-risk profiles and greater consistency and predictability of response. Here, clinical data regarding the benefits of different treatment strategies for secondary prophylaxis of VTE will be reviewed, including long-term use of LMWH, long-term oral anticoagulation with low-intensity warfarin, and treatment with novel agents including the parenterally administered pentasaccharide factor $\mathrm{Xa}$ inhibitors fondaparinux and idraparinux, as well as the first of the oral direct thrombin inhibitors, ximelagatran.

\section{Low-Molecular-Weight Heparin}

Because of the slow onset of action of warfarin, heparin therapy is typically used for 5-7 days until the full antithrombotic effect of warfarin is realized. Whilst this approach is effective in a majority of patients, warfarin therapy can be highly problematic for patients at a high risk of haemorrhagic complications, such as cancer patients. Drug interactions, malnutrition, vomiting and liver dysfunction can lead to a particularly unpredictable dose-response effect that contributes to the high rates of recurrent VTE and bleeding observed in cancer patients treated with oral anticoagulation therapy $[12,13]$. Compared with the VKAs, LMWHs have more predictable pharmacokinetic properties and require less frequent anticoagulation monitoring. They are, therefore, theoretically a more favourable treatment option for patients at a high risk of bleeding. The Randomized Comparison of Low Molecular Weight Heparin versus Oral Anticoagulation Therapy for the Prevention of Recurrent Venous Thromboembolism in Patients with Cancer (CLOT) trial recently compared the efficacy of once-daily subcutaneous administration of the LMWH dalteparin for 6 months with the conventional treatment regimen of dalteparin for 5-7 days followed by 6 months of oral anticoagulation (target INR, 2.5). The incidence of recurrent VTE was significantly reduced with long-term dalteparin treatment (8\%) compared with oral anticoagulation (16\%; hazard ratio, $0.48 ; \mathrm{p}=0.02)$, with no significant difference in the rate of major bleeding ( 6 and 4\%, respectively). Therefore, long-term LMWH treatment is a viable therapeutic option in patients at a high risk of recurrent VTE, but the subcutaneous administration of the LMWHs is a significant drawback for their use in the outpatient setting. 
Fig. 1. The ELATE trial: cumulative probability of VTE with low-intensity warfarin compared with conventional-intensity warfarin. Reproduced with permission from Kearon C et al. [15]. Copyright 2003 Massachusetts Medical Society. All rights reserved.

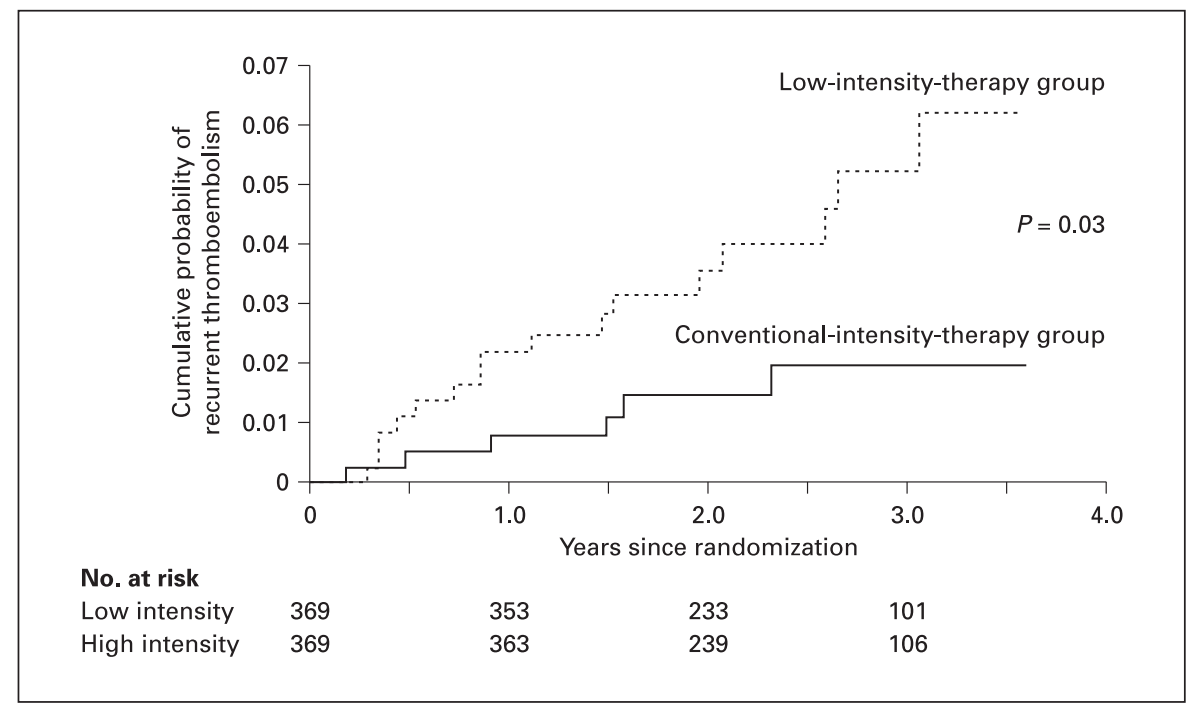

\section{Low-Intensity Warfarin}

To counter the high risks of bleeding associated with conventional-intensity warfarin (target INR, 2.0-3.0), and the need for frequent anticoagulation monitoring, long-term warfarin use at a lower intensity of INR of 1.52.0 has been proposed for the management of patients with VTE. This regimen has been evaluated in the Prevention of REcurrent VENous Thromboembolism (PREVENT) trial, in which patients with idiopathic VTE who had been receiving conventional-intensity warfarin for a median of 6.5 months were randomized to receive either low-intensity warfarin or placebo for long-term (4.3 years) secondary prevention of VTE events [14]. Low-intensity warfarin was found to be significantly more effective than placebo in reducing the risk of recurrent VTE events (2.6 and 7.2/100 person-years, respectively) and was associated with a $48 \%$ reduction in the composite end point of recurrent VTE, major bleeding and death.

These results support a role for low-intensity warfarin in the long-term secondary prevention of recurrent VTE events. However, the benefits observed in this study were not corroborated in another study. In the Extended Low-intensity Anticoagulation for Thrombo-Embolism (ELATE) trial, patients receiving conventional-intensity warfarin (target INR, 2.0-3.0) were randomized to either continue their current therapy or switch to low-intensity warfarin (target INR, 1.5-1.9) [15]. Significantly more patients on low-intensity warfarin had recurrent VTE (1.9 events/100 person-years) compared with conventional-intensity warfarin (0.7 event/100 person-years; hazard ratio, 2.8; 95\% CI, 1.1-7.0) and this reduced efficacy was not accompanied by a significant reduction in the risk of bleeding (fig. 1). In fact, slightly more patients on low- than on conventional-intensity warfarin suffered a bleeding event, although the difference was not statistically significant (1.1 vs. $0.9 / 100$ person-years; hazard ratio, $1.2 ; 95 \% \mathrm{CI}, 0.4-3.0)$. The author suggests that the reduction in efficacy seen with low-intensity warfarin may have arisen because some of the patients who switched to low-intensity warfarin did not receive a sufficiently long course of conventional-intensity warfarin before the switch. This was confirmed by analysis whereby patients were split into subgroups according to the duration of treatment before the switch, and those receiving shorter treatments were indeed found to experience higher rates of recurrence [15].

\section{Pentasaccharides}

In contrast to the heparins, which bind to several plasma components, the synthetic pentasaccharides fondaparinux and idraparinux represent the minimal binding unit of heparin, allowing them to selectively bind antithrombin. This allows rapid inhibition of factor Xa with a highly predictable pharmacokinetic profile without the need for coagulation monitoring.

Fondaparinux has been compared with the heparins as initial treatment for both deep vein thrombosis (DVT) and pulmonary embolism (PE) in the MATISSE trials $[16,17]$. These trials compared fondaparinux given sub- 
cutaneously once daily with continuous intravenous infusion of unfractionated heparin (UFH) in patients with PE [16], and with twice-daily subcutaneous injection of the LMWH enoxaparin in patients with DVT [17]. In both trials, fondaparinux or the comparator was given for at least 5 days and until VKAs caused an INR greater than 2.0.

Slightly fewer patients with $\mathrm{PE}$ receiving fondaparinux experienced recurrent VTE events (3.8\%) compared with those receiving UFH (5\%), but this difference did not reach statistical significance. Similarly, there were no significant differences in the numbers of patients experiencing bleeding events $(1.3 \%$ with fondaparinux versus $1.1 \%$ with UFH) or in the overall rate of mortality $(5.2 \%$ with fondaparinux versus $4.4 \%$ with UFH) [16]. Comparison of fondaparinux with enoxaparin in DVT patients also showed no significant differences in terms of the VTE event rate (3.9 and $4.1 \%$, respectively), the incidence of major haemorrhage (1.1 and $1.2 \%)$, and the overall mortality rate (3.8 and 3.0\%) [17]. These results suggest that fondaparinux is at least as effective and well tolerated as standard initial treatment in patients with DVT and PE, although in these trials fondaparinux did not negate the need for overlapping VKA therapy.

In contrast to fondaparinux, idraparinux has a longer half-life ( $17 \mathrm{~h}$ and $80 \mathrm{~h}$, respectively), allowing once-weekly rather than once-daily dosing, which is an important advantage for long-term outpatient treatment of VTE. The efficacy of idraparinux in the long-term prevention of VTE has been assessed in the PERSIST trial [18]. After 5-7 days of treatment with enoxaparin, patients with proximal DVT were randomized to receive one of four once-weekly doses of idraparinux $(2.5,5,7.5$ and $10 \mathrm{mg})$ or warfarin (target INR, 2.0-3.0) for 12 weeks. There was no significant difference between warfarin- and idraparinux-treated patients for the primary efficacy outcome of the composite change in thrombotic burden and clinical thromboembolic events $(p=0.4)$ [18]. There was clearly a progressive, dose-dependent increase in bleeding events among patients treated with idraparinux $(\mathrm{p}=$ 0.003 ), and only patients receiving the lowest dose of idraparinux, $2.5 \mathrm{mg}$, experienced significantly less bleeding than warfarin recipients $(p=0.003)$ [18]. It was concluded that idraparinux dosed at $2.5 \mathrm{mg}$ is as effective as warfarin for the secondary prevention of DVT with lower rates of major bleeding. Owing to these results, the 2.5-mg dose was selected for ongoing large-scale, phase III studies of idraparinux as extended treatment beyond 6 months in DVT and PE, and in stroke prevention in atrial fibrillation.

Ximelagatran in Venous

Thromboembolism

\section{Direct Thrombin Inhibitors}

Another potentially promising novel strategy for thromboprophylaxis in VTE is the use of ximelagatran, the first of the oral direct thrombin inhibitors. Ximelagatran is a candidate for use in both acute-phase and longterm secondary prevention of VTE, owing to its fast onset of action and oral administration. The efficacy and safety of ximelagatran as treatment for patients with VTE has been assessed in two large-scale, multi-centre, doubleblind, randomized phase III studies: the THRombin Inhibitor in VEnous thromboembolism (THRIVE) Treatment and THRIVE III trials. THRIVE Treatment aimed to establish whether ximelagatran is at least as efficacious and well tolerated as standard treatment over 6 months [19]. Patients with acute DVT with or without PE were randomly assigned to receive either ximelagatran $(36 \mathrm{mg}$ twice daily) or a standard therapy regimen of the LMWH enoxaparin for a minimum of 5 days followed by warfarin. Ximelagatran was given as a fixed dose without any coagulation monitoring or dose adjustments. The primary end point was confirmed new VTE events, and secondary analyses included comparison of bleeding rates, allcause mortality and the combined end point of VTE and major bleeding.

After 6 months of treatment, the rate of recurrence of VTE events was comparable between the two groups ( $2.0 \%$ with enoxaparin/warfarin vs. $2.1 \%$ with ximelagatran), demonstrating that ximelagatran is at least as effective as standard therapy (fig. 2) [19]. Additionally, ximelagatran showed non-inferiority to standard treatment in rates of major bleeding (1.3 and $2.2 \%$, respectively) and all-cause mortality (2.3 and 3.4\%). Ximelagatran was associated with an overall greater incidence of elevations in liver alanine aminotransferase (ALAT) levels to more than three times the upper limit of normal (10.2 vs. $2.2 \%$ ). However, these elevations were generally transient, occurred mainly in months 3 and 4, were asymptomatic in terms of liver function and resolved spontaneously whether treatment was continued or discontinued [19]. Therefore, this study demonstrated that oral ximelagatran is at least as efficacious and well tolerated as standard therapy for the acute treatment of VTE over 6 months [19].

The THRIVE III trial expanded on these results by assessing the potential of ximelagatran for long-term secondary prevention beyond 6 months, at which point anticoagulation with current agents is often stopped [20]. Patients with DVT and/or PE who had undergone treatment for 6 months with a VKA were randomized to receive ximelagatran $24 \mathrm{mg}$ twice daily without coagulation 
Fig. 2. THRIVE treatment: estimated treatment differences between ximelagatran and standard therapy with enoxaparin/warfarin after 6 months of treatment [19]. ITT = Intent to treat; $\mathrm{OT}=$ on treatment.
Fig. 3. THRIVE III: estimated cumulative risk of a recurrent VTE event during 18 months of treatment with ximelagatran versus placebo. $\mathrm{HR}=$ Hazard ratio. Reproduced with permission from Schulman S et al. [20]. Copyright 2003 Massachusetts Medical Society. All rights reserved.
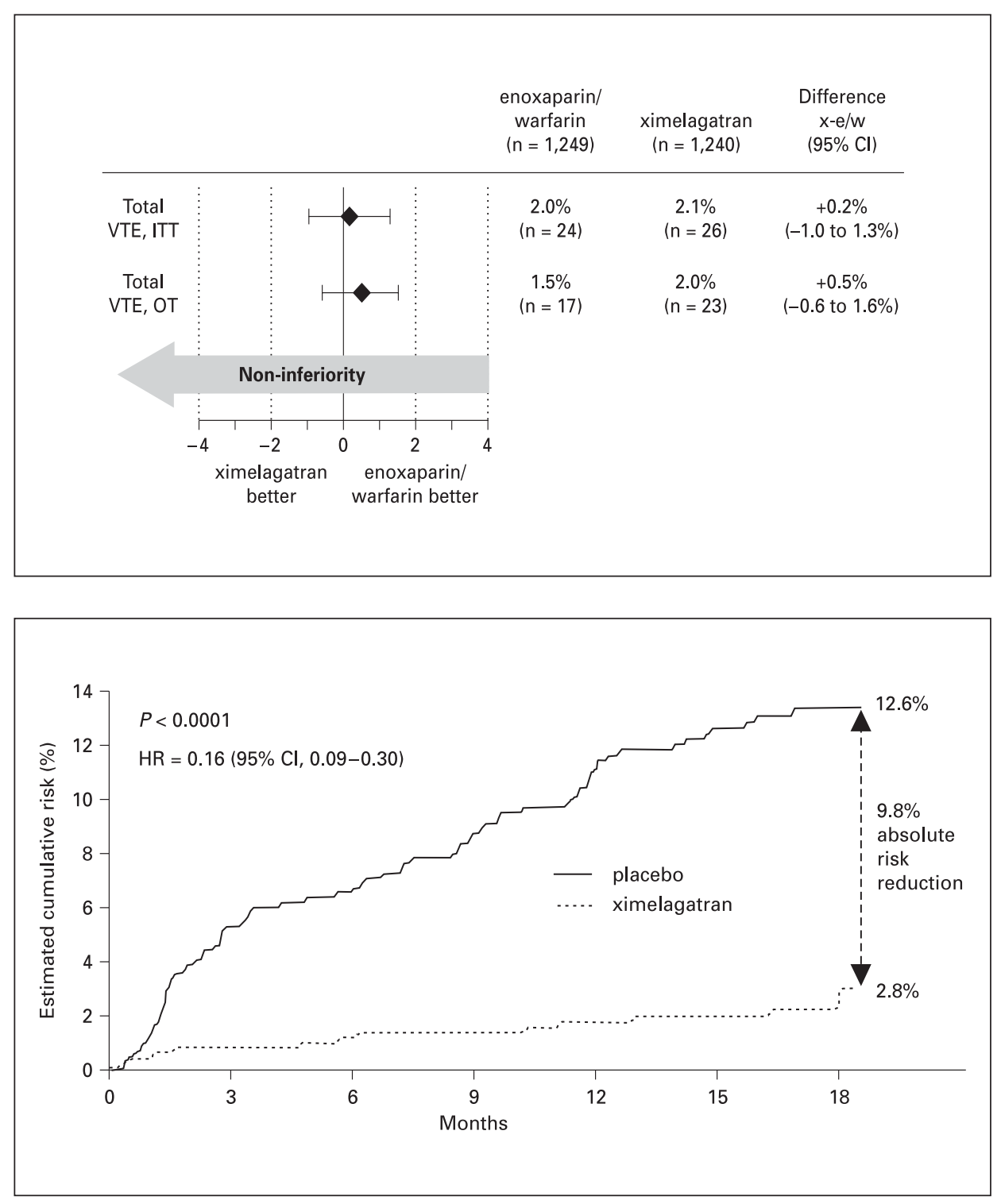

monitoring, or placebo, for 18 months. The primary objective of the trial was to assess the time to a confirmed new VTE event, with secondary objectives including the assessment of mortality risk and other safety parameters, particularly bleeding episodes.

The estimated cumulative risk of VTE events was dramatically reduced among patients treated with ximelaga$\operatorname{tran}(2.8 \%)$ compared with those who stopped anticoagulation at 6 months $(12.6 \%$; risk reduction, $9.8 \% ; \mathrm{p}<0.001$; fig. 3).

Analysis of major and minor bleeding revealed no significant difference in the estimated cumulative risk of bleeding events ( $p=0.17$; fig. 4 ), and all-cause mortality occurred in $1 \%$ of patients in each treatment group. More patients receiving ximelagatran experienced increased levels of ALAT, with an incidence of more than three times the upper limit of normal of $6.0 \%$ in the ximelagatran group compared with $1.0 \%$ in the placebo group (cumulative risk of 6.4 and $1.2 \%$, respectively; $p<0.001$ ) [20]. Again, these elevations were seen mainly in months 3 and 4 of treatment, were typically transient and asymptomatic in nature, and resolved spontaneously with or without treatment discontinuation.

Therefore, the THRIVE III study demonstrated that ximelagatran at a dose of $24 \mathrm{mg}$ twice daily significantly reduces the risk of symptomatic recurrent VTE events, 
Fig. 4. THRIVE III: estimated cumulative risk of a major or minor bleeding event during 18 months of treatment with ximelagatran versus placebo. Reproduced with permission from Schulman S et al. [20]. Copyright 2003 Massachusetts Medical Society. All rights reserved.

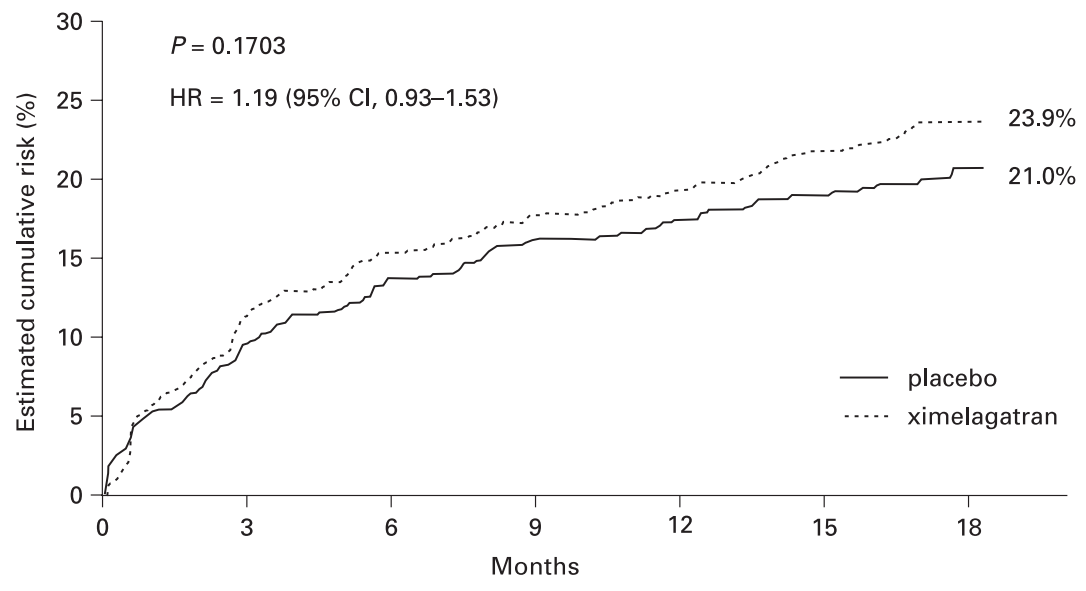

with an incidence of bleeding similar to that seen in the placebo group. Thus, ximelagatran possesses a favourable benefit-risk profile for long-term secondary prevention of VTE events.

\section{Conclusions}

Ximelagatran, by overcoming many of the limitations associated with current treatment strategies, offers considerable potential for the improved management of VTE. Unlike warfarin, orally administered ximelagatran can be given immediately upon diagnosis owing to its fast onset of action and can be used effectively in both acutephase treatment and long-term secondary prevention of VTE. Furthermore, the fixed dosing regimen and predictable pharmacokinetic profile relieves the burden of management associated with regular coagulation monitoring and dose adjustments. Thus, the improved benefit-risk profile of ximelagatran has the potential to extend the duration of therapy and maximize the potential of highly effective anticoagulation in the treatment and secondary prevention of VTE.

\section{References}

1 Prandoni P, Lensing AW, Cogo A, Cuppini S, Villalta S, Carta M, Cattelan AM, Polistena P, Bernardi E, Prins MH: The long-term clinical course of acute deep venous thrombosis. Ann Intern Med 1996;125:1-7.

$\checkmark 2$ Hirsh J: The optimal duration of anticoagulant therapy for venous thrombosis. N Engl J Med 1995;332:1710-1711.

\3 Hyers TM, Agnelli G, Hull RD, Morris TA, Samama M, Tapson V, Weg JG: Antithrombotic therapy for venous thromboembolic disease. Chest 2001;119:176S-193S.

$\checkmark 4$ Schulman S, Rhedin AS, Lindmarker P, Carlsson A, Larfars G, Nicol P, Loogna E, Svensson E, Ljungberg B, Walter $\mathrm{H}$ : A comparison of six weeks with six months of oral anticoagulant therapy after a first episode of venous thromboembolism. Duration of Anticoagulation Trial Study Group. N Engl J Med 1995;332:16611665.
Schulman S, Granqvist S, Holmstrom M, Carlsson A, Lindmarker P, Nicol P, Eklund SG, Nordlander S, Larfars G, Leijd B, Linder O, Loogna E: The duration of oral anticoagulant therapy after a second episode of venous thromboembolism. The Duration of Anticoagulation Trial Study Group. N Engl J Med 1997;336:393-398.

6 Fihn SD, Callahan CM, Martin DC, McDonell MB, Henikoff JG, White RH: The risk for and severity of bleeding complications in elderly patients treated with warfarin. The National Consortium of Anticoagulation Clinics. Ann Intern Med 1996;124:970-979.
7 Beyth RJ, Quinn LM, Landefeld CS: Prospective evaluation of an index for predicting the risk of major bleeding in outpatients treated with warfarin. Am J Med 1998;105:91-99.

$\checkmark 8$ McMahan DA, Smith DM, Carey MA, Zhou $\mathrm{XH}$ : Risk of major hemorrhage for outpatients treated with warfarin. J Gen Intern Med 1998; 13:311-316.

9 Ansell J, Hirsh J, Dalen J, Bussey H, Anderson D, Poller L, Jacobson A, Deykin D, Matchar D: Managing oral anticoagulant therapy. Chest 2001;119:22S-38S.

10 Landefeld CS, Goldman L: Major bleeding in outpatients treated with warfarin: Incidence and prediction by factors known at the start of outpatient therapy. Am J Med 1989;87:144152. 
11 Agnelli G: Current issues in anticoagulation. Pathophysiol Haemost Thromb 2004;34 (suppl 1):2-9.

12 Hutten BA, Prins MH, Gent M, Ginsberg J, Tijssen JG, Buller HR: Incidence of recurrent thromboembolic and bleeding complications among patients with venous thromboembolism in relation to both malignancy and achieved international normalized ratio: A retrospective analysis. J Clin Oncol 2000;18: 3078-3083.

- 13 Prandoni P, Lensing AW, Piccioli A, Bernardi E, Simioni P, Girolami B, Marchiori A, Sabbion P, Prins MH, Noventa F, Girolami A: Recurrent venous thromboembolism and bleeding complications during anticoagulant treatment in patients with cancer and venous thrombosis. Blood 2002; 100:3484-3488.

14 Ridker PM, Goldhaber SZ, Danielson E, Rosenberg Y, Eby CS, Deitcher SR, Cushman M, Moll S, Kessler CM, Elliott CG, Paulson R, Wong T, Bauer KA, Schwartz BA, Miletich JP, Bounameaux H, Glynn RJ: Long-term, low-intensity warfarin therapy for the prevention of recurrent venous thromboembolism. N Engl J Med 2003;348:1425-1434.
15 Kearon C, Ginsberg JS, Kovacs MJ, Anderson DR, Wells P, Julian JA, MacKinnon B, Weitz JI, Crowther MA, Dolan S, Turpie AG, Geerts W, Solymoss S, van Nguyen P, Demers C, Kahn SR, Kassis J, Rodger M, Hambleton J, Gent M: Comparison of low-intensity warfarin therapy with conventional-intensity warfarin therapy for long-term prevention of recurren venous thromboembolism. N Engl $\mathrm{J}$ Med 2003;349:631-639.

16 Buller HR, Davidson BL, Decousus H, Gallus A, Gent M, Piovella F, Prins MH, Raskob G, van den Berg-Segers AE, Cariou R, Leeuwenkamp O, Lensing AW: Subcutaneous fondaparinux versus intravenous unfractionated heparin in the initial treatment of pulmonary embolism. N Engl J Med 2003;349:16951702 .
17 Buller HR, Davidson BL, Decousus H, Gallus A, Gent M, Piovella F, Prins MH, Raskob G, Segers AE, Cariou R, Leeuwenkamp O, Lensing AW: Fondaparinux or enoxaparin for the initial treatment of symptomatic deep venous thrombosis: A randomized trial. Ann Intern Med 2004; 140:867-873.

18 PERSIST Investigators: A novel long-acting synthetic factor Xa inhibitor (SanOrg34006) to replace warfarin for secondary prevention in deep vein thrombosis. A Phase II evaluation. J Thromb Haemost 2004; 2:47-53.

19 Francis CW, Ginsberg JS, Berkowitz SD, Bounameaux H, Davidson BL, Eriksson H, Fiessinger J-N, Huisman MV, Lundstrom T, Nystrom $\mathrm{P}$, the THRIVE Treatment Study Investigators: Efficacy and safety of the oral direct thrombin inhibitor ximelagatran compared with current standard therapy for acute, symptomatic deep vein thrombosis, with or without pulmonary embolism: The THRIVE Treatment Study. Blood 2003;102:abstract 7.

20 Schulman S, Wåhlander K, Lundström T, Clason SB, Eriksson H, THRIVE III Investigators: Secondary prevention of venous thromboembolism with the oral direct thrombin inhibitor ximelagatran. N Engl J Med 2003;349:17131721. 